\title{
Towards off-the-shelf networks
}

Synthetic biologists dream of constructing gene expression networks with predictable functions. However, they come up against a frustrating problem: because the assembly parts (ORFs and control regions) are poorly characterized and limited in number, each new circuit has to be painstakingly tweaked until it behaves as intended. To overcome this hurdle Ellis and colleagues have generated and characterized a library of components, and then used computer modelling to inform how these components - in this case, promoters - should be assembled for particular uses.

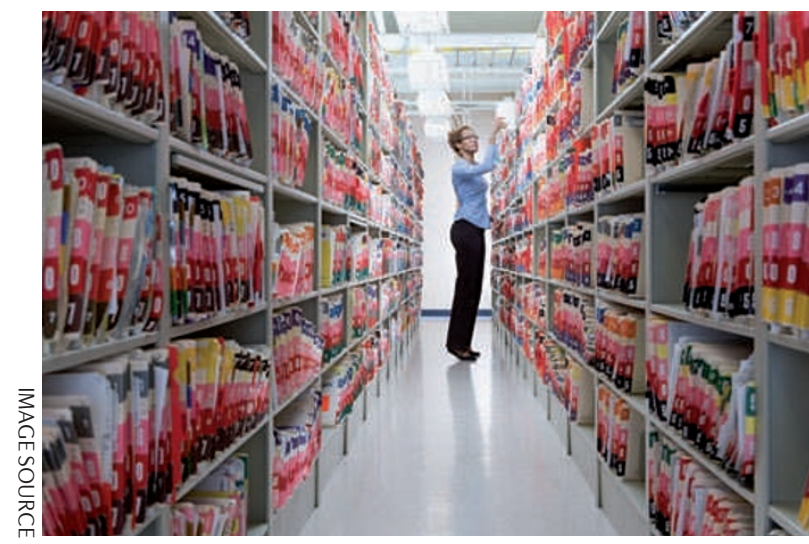

To produce the promoter library a set of Saccharomyces cerevisiae promoters that can be regulated by TetR was generated using a synthesis protocol that specifically alters nonessential sequences. The library components were then classified by their expression output - as inferred from a reporter gene, EGFP — depending on the concentration of TetR.

An in silico prediction method was used to select which of the 20 promoters would be the most appropriate to use in any particular type of circuit. For example, in a feed-forward loop two repressors (LacI and TetR) feed onto an output gene, but with TetR also inhibiting LacI. The challenge was to select, from the library, the LacI promoter that would yield a predicted expression landscape in response to varying concentrations of TetR and LacI inducers. The input-output landscape of the three experimentally constructed networks correlated well with computational predictions.

The approach was also effective when applied to a more complicated circuit, a genetic timer: here, expression levels switch (toggle) from one state to another depending on the relative concentration of opposing repressor molecules. Two libraries of promoters were used in this application, and the model was derived by combining first principles with a single reconstructed circuit; however, as before, the experimental circuits behaved as expected.

The predictions associated with the genetic timer were applied to control the timing of activation of a yeast gene that triggers the clumping and sedimentation of yeast cells. This process allows cells to be removed easily from fermented liquid, and is therefore of interest in beer and wine production.

This off-the-shelf strategy promises to accelerate the rate of progress of synthetic biology by removing the fiddly adjustments that currently hamper the post-construction stage. And promoters are just the beginning the same principles can be extended to other biomolecular components, such as RNAs or proteins.

Tanita Casci

ORIGINAL RESEARCH PAPER Ellis, T. et al.

Diversity-based, model-guided construction of synthetic gene networks with predicted functions. Nature Biotech. 19 Apr 2009 (doi:10.1038/nbt.1536) 